PROCEEDINGS OF THE

AMERICAN MATHEMATICAL SOCIETY

Volume 127, Number 11, Pages 3125-3130

S 0002-9939(99)05426-X

Article electronically published on July 12, 1999

\title{
NON-SEMISTABLE ARAKELOV BOUND AND HYPERELLIPTIC SZPIRO RATIO FOR FUNCTION FIELDS
}

\author{
KHAC VIET NGUYEN \\ (Communicated by Ron Donagi) \\ Dedicated to the memory of Professor K. Kodaira
}

\begin{abstract}
We prove a complex function field analogue of Szpiro's conjecture for hyperelliptic curves and some applications. The cases of function fields of positive characteristic and number fields are discussed briefly.
\end{abstract}

\section{INTRODUCTION}

1.1. Let $K$ be a global function field of characteristic zero, i.e. $K$ is a field of functions on a non-singular complete curve $C$ with an algebraically closed field $k$ of characteristic zero as the field of constants. For a smooth algebraic curve $Y$ of genus $g \geq 1$ defined over $K$ one can uniquely associate a smooth projective fibred surface $f: X \rightarrow C$ with generic fibre $Y / K$ and such that degenerate fibres contain no $(-1)$-curves - its minimal model. In the case of an elliptic curve $E / K(g=1)$ the following statement is well known (due to Kodaira-Shioda).

The function field analogue of Szpiro's conjecture. ([Sh, Prop. 2.8], cf . [Hi-Si]) Let $E / K$ be a non-constant elliptic curve. Then

$$
\operatorname{deg} \mathcal{D}_{E / K} \leq 6\left(2 g(C)-2+\operatorname{deg} \mathcal{N}_{E / K}\right)
$$

where $\mathcal{D}_{E / K}, \mathcal{N}_{E / K}$ denote the minimal discriminant and conductor divisors of $E / K$ respectively.

The aim of this note is to generalize (1.2) for hyperelliptic curves over function fields. In the hyperelliptic case it is natural to use the following notion $\mathcal{D}_{Y / K}$ of the minimal discriminant divisor ( $c f$. [Ma], [U]). Since in this case $f: X \rightarrow C$ is a double covering of a ruled surface $\pi: X^{\prime} \rightarrow C$, there exists an open subset $C^{0}$ in $C$ such that

(i) $\pi^{-1}\left(C^{0}\right) \simeq \mathbb{P}^{1} \times C^{0}$;

(ii) $f^{-1}\left(C^{0}\right)$ can be identified with the closure of $\left\{(x, y, t) \in \mathbb{A}^{1} \times \mathbb{A}^{1} \times C\right.$ : $\left.y^{2}=\varphi(x, t)\right\}$, where $x$ is an inhomogeneous coordinate of $\mathbb{P}^{1}$ and $\varphi$ is a polynomial in $x$ of degree $2 g+1$, or $2 g+2$ with coefficients in the rational function field of

Received by the editors June 12, 1997.

1991 Mathematics Subject Classification. Primary 11G30, 14H05.

Key words and phrases. Non-semistable, hyperelliptic, Arakelov bound, Szpiro ratio.

The research was partially supported by the National Natural Science Basic Research Foundation of Vietnam. 
$C^{0}$. Letting $\Delta(\varphi)$ be the discriminant of $\varphi$ as a polynomial in $x$ and regarding $\omega_{i}:=x^{i-1} \frac{d x}{y}, i=1,2, \ldots, g$, as rational sections of $f_{*} \omega_{X / C}$, the section

$$
D:=\Delta(\varphi)^{g}\left(\omega_{1} \wedge \omega_{2} \wedge \ldots \wedge \omega_{g}\right)^{\otimes 4(2 g+1)},
$$

independent of the choice of $\left(X^{\prime}, C^{0}, x, y, \varphi\right)$, defines an element of

$$
H^{0}\left(C,\left(\bigwedge^{g} f_{*} \omega_{X / C}\right)^{\otimes 4(2 g+1)}\right) .
$$

Set

$$
\mathcal{D}_{Y / K}:=\sum_{t \in C} \frac{\operatorname{ord}_{t}(D)}{g}[t] .
$$

Our next task is to define the notion of the conductor divisor of $Y / K$. In the elliptic case the conductor divisor of $E / K$ is nothing but the conductor divisor of the associated Jacobian fibration. However in the higher genus case it may happen that $\operatorname{Pic}^{0}(X / C)$ has good reduction at $t$ but $f$ itself - not, in other words, the dual graph of fibre $X_{t}$ over $t$ - is a tree with (at least two) smooth irreducible components. There are two main ingredients supporting the approach here: non-semi-stable variant of Arakelov's bound (see section 2.) together with Matsusaka's inequalities between local degrees $d_{t}(X)$ and local Euler numbers $e_{t}(X)$ ([Ma, Theorem 4.0.1]) which lead us to the following definition.

Definition 1.4. The conductor divisor of $Y / K$ is defined to be

$$
\mathcal{N}_{Y / K}:=\sum_{t \in C}\left(\operatorname{ord}_{t}\left(\mathcal{D}_{Y / K}\right)-n_{t}+1\right)[t]
$$

where $n_{t}$ denotes the number of irreducible components in the fibre $X_{t}$ over $t$.

It should be noted that in (1.3) and (1.5) the sum is taken over just finitely many non-zero terms.

Definition 1.6. We define by analogy with elliptic curves the Szpiro ratio as

$$
\sigma_{Y / K}:=\frac{\operatorname{deg} \mathcal{D}_{Y / K}}{\operatorname{deg} \mathcal{N}_{Y / K}}
$$

so that (1.2) is equivalent to

$$
\sigma_{E / K}^{-1} \geq \frac{1}{6}\left(1-\frac{g(C)-1}{h_{K}(E)}\right)
$$

where $h_{K}(E)$ is the height of $E$ which in this case is just $\frac{1}{12} \operatorname{deg} \mathcal{D}_{E / K}(c f$. [Hi-Si]).

We have the following bounds which are a generalization of (1.2) and (1.8) (cf. Theorem 3.2):

$$
g \operatorname{deg} \mathcal{D}_{Y / K} \leq 2(2 g+1)\left(g(2 g(C)-2)+2 g_{0}+\operatorname{deg} \mathcal{N}_{Y / K}\right)
$$

where $g_{0}:=\operatorname{dim}$ of the fixed part of $\operatorname{Pic}^{0}(X / C)$; or equivalently (with a suitable convention in the locally trivial case)

$$
\sigma_{Y / K}^{-1} \geq \frac{g}{2(2 g+1)}\left(1-\frac{g(C)-1+g_{0} / g}{h_{K}(Y)}\right) .
$$


Here the term (again by analogy with elliptic curves)

$$
h_{K}(Y):=\frac{1}{4(2 g+1)} \operatorname{deg} \mathcal{D}_{Y / K}=\frac{d}{g}
$$

with $d:=\operatorname{deg} f_{*} \omega_{X / C}$ should be called the height of $Y / K$.

In fact, as was remarked above and as will be shown in section 3., these bounds are immediate consequences of Matsusaka's inequalities and a non-semi-stable variant of Arakelov's bound for the degree $d=\operatorname{deg} f_{*} \omega_{X / C}$ which will be given in the next section. As another application we turn to the problem of classifying fibrations of curves over $\mathbb{P}^{1}$ with small number of singular fibres $(c f$. [N2]). In closing the note we briefly discuss the function field case of positive characteristic and the number field case.

\section{NON-SEMI-STABlE VARIANT OF ARAKELOV'S BOUND}

2.1. In this section we first prepare more notation and facts which we shall need in the following. For a relatively minimal fibration of curves $f: X \rightarrow C$ let $S$ (resp. $S_{1}$ ) be the set of points of (resp. semi-stable) degeneracy on $C$, and let $s, s_{1}$ denote their cardinalities, i.e. $s$ is the total number of singular fibres and $s_{1}$ is the number of semi-stable singular fibres of $f$. As usual, $n_{t}$ denotes the number of irreducible components in the fibre $X_{t}$ and $e_{t}(X):=\chi\left(X_{t}\right)-(2-2 g)$ is the local Euler number. Also a purely technical calculation of local Euler numbers using the local intersection theory on $X_{t}$ shows that there are the following bounds for the "tame" conductor $\varepsilon_{t}(X):=e_{t}(X)-n_{t}+1(c f .[\mathrm{N} 2$, Lemma 1]):

$$
g-g\left(\widetilde{X}_{t}\right) \leq \varepsilon_{t}(X) \leq 2\left(g-g\left(\widetilde{X}_{t}\right)\right)
$$

where $g\left(\widetilde{X}_{t}\right)$ denotes the genus of the normalization of $\left(X_{t}\right)_{\text {red }}$. Moreover the equality on the left holds for semi-stable fibres $X_{t}$.

Theorem 2.3. Let $f: X \rightarrow C$ be a relatively minimal fibration of curves. In the notation above we have the following upper bound for the degree $d$ :

$$
d \leq\left(g-g_{0}\right)\left(g(C)-1+s-\frac{s_{1}}{2}\right)+g_{0} g(C) .
$$

Proof. Recall first the arguments similar to those in [N1] by using the following standard formulae:

$$
\begin{aligned}
& p_{g}=d+g(g(C)-1)+g_{0}, \\
& \rho=2+\sum_{t \in C}\left(n_{t}-1\right)+r, \\
& c_{2}(X)=4(g-1)(g(C)-1)+\sum_{t \in C} e_{t}(X)
\end{aligned}
$$

where $r$, as usual, is the virtual Mordell-Weil rank and $\rho$ denotes the Picard number of $X$.

Further by definition

$$
\chi(X)=2-4 q(X)+h^{1,1}+2 p_{g} .
$$

Combining all these together one gets

$$
d=g(g(C)-1)+g_{0}+\frac{1}{2}\left(\sum_{t \in S} \varepsilon_{t}(X)-\rho^{\prime}-r\right)
$$


where $\rho^{\prime}:=h^{1,1}-\rho$ is the number of "transcendental" $(1,1)$-cycles which is a non-negative integer in view of the Lefschetz $(1,1)$-theorem. Thus we deduce from $(2.2)$ and $(2.5)$

$$
d \leq\left(g-g_{0}\right)\left(q-1+s-\frac{s_{1}}{2}\right)+g_{0} g(C)-\sum_{t \in S \backslash S_{1}}\left(g\left(\tilde{X}_{t}\right)-g_{0}\right)-\frac{1}{2} \sum_{t \in S_{1}}\left(g\left(\tilde{X}_{t}\right)-g_{0}\right) .
$$

Now estimate $(2.4)$ can be obtained by remarking that $g\left(\widetilde{X}_{t}\right) \geq g_{0}(c f$. [N1, A.2.3]) $\forall t \in C$.

Remark 2.6. It is easy to see that for semi-stable fibrations $f: X \rightarrow C$ one has the following bound (cf. [A], [N1, A.2.4]):

$$
d \leq\left(g-g_{0}\right)\left(g(C)-1+\frac{s}{2}\right)+g_{0} g(C) .
$$

In fact if we assume that $f$ is non-isotrivial, then by using the Variation of Hodge Structure the term $g_{0} g(C)$ from the right-hand side of (2.7) can be removed. It should be remarked also that as a corollary to (2.4) one gets (1.2) for the elliptic case mentioned at the beginning of the note.

2.8. We discuss briefly a use of bound (2.4) to the problem of classifying locally non-trivial fibrations $f: X \rightarrow \mathbb{P}^{1}$ with $s \leq 3$ that was begun in [N2]. In fact by using (2.4) one may select several extremal cases. For example, if $s=2$, or $s=3$ and $s_{1}=2$, or $r=2 g$, then equality attains in (2.4): $d=g-g_{0}$ (a bit more work shows that we have in fact $g_{0}=0 ; c f$. [N2]). On the other hand from the formula of Ogg-Shafarevich-Grothendieck for $f$ (loc. cit.) one can see easily that $s=3$ implies: $0 \leq p_{g} \leq g$ and $0 \leq r \leq 2 g$. It should be noted that the extremal possibility $p_{g}=g$ is also of interest. In this case either $X$ is a $K 3$ surface $(g=1)$, or $X$ admits a "general" elliptic fibration, or $X$ is a minimal surface of general type. I do not know examples of the last two types.

\section{Hyperelliptic fibrations and the SzPiro Ratio}

3.1. In this section let $f: X \rightarrow C$ be a hyperelliptic fibration. Recall that $K=$ $k(C)$ and $Y=X \times_{C} \operatorname{Spec}(K)$. Our aim is to prove the following theorem.

Theorem 3.2. Notation being as above

$$
g \operatorname{deg} \mathcal{D}_{Y / K} \leq 2(2 g+1)\left(g(2 g(C)-2)+2 g_{0}+\operatorname{deg} \mathcal{N}_{Y / K}\right)
$$

or equivalently

$$
\sigma_{Y / K}^{-1} \geq \frac{g}{2(2 g+1)}\left(1-\frac{g(C)-1+g_{0} / g}{h_{K}(Y)}\right) .
$$

As was remarked in the Introduction in the context here the term

$$
h_{K}(Y):=\frac{1}{4(2 g+1)} \operatorname{deg} \mathcal{D}_{Y / K}=\frac{d}{g}
$$

plays a similar role as the height of $E / K$ in the elliptic case. Note that (3.4) should be understood suitably for locally trivial fibrations. 
3.5. Recall that the local degrees $d_{t}(X)$ are defined to be

$$
d_{t}(X):=\frac{1}{4(2 g+1)} \operatorname{ord}_{t}(D)
$$

where $D$ is the section from 1 . The point here is to show that the invariants $d_{t}(X)$ are non-negative, so that $D$ is actually a regular section. More precisely, one has ([Ma, Theorem 4.0.1])

$$
\frac{g}{4(2 g+1)} e_{t}(X) \leq d_{t}(X)
$$

and as shown in [N1] with equality if and only if $X$ is the canonical resolution over $t$ of a double covering of a geometrically ruled surface with rational double points as its singularities. Therefore in view of (1.5) and (2.2) one can see that $\operatorname{ord}_{t}\left(\mathcal{N}_{Y / K}\right)=g-g\left(\widetilde{X}_{t}\right)$ only in the case of $X_{t}$ semi-stable. On the other hand by the definition of $D$ clearly $\sum_{t \in C} d_{t}(X)=d$, and so

$$
g \operatorname{deg} \mathcal{D}_{Y / K}=4(2 g+1) d .
$$

Now we are ready to complete the proof of Theorem 3.2. First note that an easy arithmetic shows the obvious equivalence of (3.3) and (3.4). Next from (3.6), (1.3) and (1.5) it follows that

$$
\operatorname{deg} \mathcal{N}_{Y / K} \geq \sum_{t \in S} \varepsilon_{t}(X)
$$

Combining (2.5), (3.7) and (3.8) one gets (3.3).

\section{REMARKS}

4.1. We consider the case of a function field of characteristic $p$. For elliptic curves everything in 1. works well ( $c f$. [G-S] and references therein). In the higher genus case we have difficulty with defining the notion of the minimal discriminant divisor as in 1., because $a$ priori the section $D$ may not be regular (this still works if $g=2$ and $p \neq 2$, as shown in [U] by checking case by case with the aid of the complete classification of singular fibres for this case). However one can have a bound of type (2.7) in the semi-stable case of positive characteristic.

Theorem 4.2. Let $f: X \rightarrow C$ be a non-isotrivial semi-stable fibration with inseparability exponent $e$. Then

$$
d<2 p^{e} g^{2}\left(g(C)-1+\frac{s}{2}\right) .
$$

Proof. It follows directly from [Sz, exposé III, Théorèm 3] and Moriwaki's version of the Cornalba-Harris-Xiao inequality ([Mo] $)$.

In positive characteristic, as usual, the non-semi-stable case is very difficult ( $c f$. [Sz, exposé III]). Presumably in the genus 2 case by using [U] it could be shown that (4.3) is a version of Szpiro's conjecture. For example, one can guess that the definitions (1.3), (1.5) are still good by testing on the Moret-Bailly families ([Sz, exposés VII-VIII]). 
4.4. In the number field case it should be very interesting to have a precise formulation of the higher genus version of Szpiro's conjecture using ideas along these lines. Because of the reasons given above the most worthwhile thing to do is to formulate the genus 2 case clearly and carefully which I do hope to come back to in a future paper. Finally I would mention that in general there is a completely different approach inspired by Ogg's notion of the minimal discriminant of curves of genus $2(c f .[\mathrm{L}])$, but I could not see a precise function field analogue in this approach.

\section{ACKNOWLEDGEMENTS}

I would like to thank Professors V. A. Iskovskikh, A. N. Parshin, T. Shioda, H. Esnault and E. Viehweg for encouragement and valuable suggestions.

\section{REFERENCES}

[A] Arakelov, S. Yu., Families of algebraic curves with fixed degeneracy, Math. USSR Izv., v.5 (1971), 1277-1302. MR 48:298

[G-S] Goldfeld D., Szpiro L., Bounds for the order of the Tate-Shafarevich group, Comp. Math., 97 (1995), 71-87. MR 97a:11102

[Hi-Si] Hindry, M., Silverman, J. H., The canonical height and integral points on elliptic curves, Invent. Math., 93 (1988), 419-450. MR 89k:11044

[L] Lockhart, P., On the discriminant of a hyperelliptic curve, Trans. of AMS, 342-2(1994), 729-752. MR 94f: 11054

[Ma] Matsusaka, S., Some numerical invariants of hyperelliptic fibrations, J. Math. Kyoto Univ., 30-1 (1990), 33-57. MR 91a:14016

[Mo] Moriwaki, A., Bogomolov conjecture over function fields for stable curves with only irreducible fibers, Comp. Math., 105 (1997), 125-140. MR 98d:14036

[N1] Nguyen, K. V., On Beauville's conjecture and related topics, J. of Math. of Kyoto Univ., 35-2 (1995), 275-298. MR 96m:14032b

[N2] Nguyen, K. V., On families of curves over $\mathbb{P}^{1}$ with small number of singular fibres, C. R. Acad. Sci. Paris, 326 (1998), Série I, 459-463. CMP 99:02

[Sh] Shioda, T., On elliptic modular surfaces, J. Math. Soc. Japan, 24 (1972), 20-59. MR $\mathbf{5 5 : 2 9 2 7}$

[Sz] Szpiro, L., Séminaire sur les pinceaux de courbes de genre au moins deux, Astérisque, v.86 (1981). MR 83c:14020

[U] Ueno, K., Discriminants of curves of genus 2 and arithmetic surfaces, Algebraic Geometry and Commutative Algebra in Honor of M. Nagata (1987), 749-770. MR 90a:14040

Institute of Mathematics, P.O. Box 631, Bo Ho, 10000, Hanoi, Vietnam

E-mail address: nkviet@thevinh.ncst.ac.vn 